\title{
A ATUAÇÃO DO PSICÓLOGO NA ESCOLA DE ENSINO FUNDAMENTAL: MODALIDADES DE TRABALHO
}

\author{
EL DESEMPEÑO DEL PSICÓLOGO EN LA ESCUELA DE EDUCACIÓN \\ FUNDAMENTAL: MODALIDADES DE TRABAJO
}

\author{
PSYCHOLOGIST'S PERFORMANCE IN ELEMENTATRY SCHOOL: WORKING \\ MODES
}

\author{
Rafaela Fava de QUEVEDO ${ }^{1}$
}

RESUMO: Um psicólogo no contexto escolar é facilitador de possibilidades, especialmente no Ensino Fundamental. Diante isso, o objetivo desse estudo foi de investigar a atuação do psicólogo na escola de ensino fundamental, pontuando as modalidades de trabalho que estão sendo executadas atualmente. Realizou-se uma revisão sistemática contemplando uma busca nas bases de dados Biblioteca Virtual em Saúde, PubMed Central, Scielo, Directory of Open Access Journals e Directory of Open Access Repositories. Foram analisados oito artigos, considerando as características dos participantes e as modalidades de intervenções, bem como os resultados desses trabalhos. A quantidade de trabalhos encontrados demarca uma fragilização de relatos de experiência dos profissionais da psicologia, sendo que essa modalidade de trabalho é pouco valorizada pela comunidade científica. Sugere-se que sejam desenvolvidos trabalhos na modalidade de relatos de experiência para a divulgação do conhecimento sobre intervenções de profissionais da psicologia e desenvolvimento das práticas psicológicas em contexto escolar.

PALAVRAS-CHAVE: Psicologia educacional. Escola. Intervenções. Revisão sistemática.

RESUMEN: Un psicólogo escolar es un facilitador de posibilidades, especialmente en la escuela primaria. Ante esto, el objetivo de este estudio fue investigar el papel del psicólogo en la escuela primaria, puntuando los tipos de trabajo que se realizan actualmente. Se realizó una revisión sistemática contemplando una búsqueda en la Biblioteca Virtual en Salud, PubMed Central, Scielo, el Directorio de Revistas de Acceso Abierto y el Directorio de bases de datos de Repositorios de Acceso Abierto. Se analizaron ocho artículos, considerando las características de los participantes y las modalidades de las intervenciones, así como los resultados de estos trabajos. La cantidad de trabajo encontrado marca un debilitamiento de los informes de experiencia de profesionales en psicología, y este tipo de trabajo es poco valorado por la comunidad científica. Se sugiere que el trabajo se desarrolle en forma de informes de experiencia para la difusión del conocimiento de las intervenciones por parte de profesionales en psicología y el desarrollo de prácticas psicológicas en el contexto escolar.

PALABRAS CLAVE: Psicología Educacional. Colegio. Intervenciones. Revisión sistemática.

${ }^{1}$ Universidade do Vale do Rio dos Sinos (UNISINOS), São Leopoldo - RS - Brasil. Mestre em Psicologia Clínica. ORCID: http://orcid.org/0000-0001-8968-8064. E-mail: rafaelafaq@msn.com 
ABSTRACT: A psychologist in the school context facilitates possibilities, especially in elementary school. Given this, the objective of this study was to investigate the performance of the psychologist in elementary school, punctuating the modalities of work that are being performed. A systematic review was made to search the databases Virtual Health Library, PubMed Central, Scielo, Directory of Open Access Journals and Directory of Open Access Repositories. Keywords: psychology, school, experience report. Thus, eight articles were analyzed, considering the characteristics of the participants and the modalities of interventions, as well as the results from these works. The amount of work found marks a weakening of experience reports of psychology professionals, and this modality of work ends up being little valued by the scientific community. It is suggested that the construction of experience reports be made for the knowledge on interventions of psychology professionals contribute further development of psychological practices in the school context.

KEYWORDS: Educational psychology. School. Interventions. Systematic review.

\section{Introdução}

A atuação do psicólogo no campo da educação pode ser apresentada como recente no Brasil. A psicologia carrega a marca da atuação clínica, enquanto, por outro lado, existem movimentos do Conselho Federal de Psicologia (CFP) para que o cargo de psicólogo seja obrigatório nas escolas públicas do país. A justificativa é de que o psicólogo atuando em ambiente escolar venha a promover a saúde mental, consequentemente atenuando potenciais riscos que possam emergir na vida adulta.

O psicólogo no cenário da educação se caracterizou por muito tempo em ajustar os alunos ao modelo de escola e sociedade, bem como auxiliar os alunos que apresentavam problemas de aprendizagem e de comportamento (EVANGELISTA; AMARAL, 2017; BARBOSA; MARINHO-ARAÚJO, 2010; OLIVEIRA; MARINHO-ARAÚJO, 2009, VALLE, 2003). Atualmente, a participação do psicólogo na escola, apresenta uma postura mais em torno a atuação preventiva e relacional, valorizando a todos os agentes escolares e sua saúde mental (OLIVEIRA; MARINHO-ARAÚJO, 2009). Dessa forma, o psicólogo escolar contribui na valorização dos seres humanos que atuam num mesmo lugar, algo considerado como uma atuação independente da consolidada prática clínica (BARBOSA; MARINHO-ARAÚJO, 2010).

A escola se caracteriza como um espaço demarcado pelos princípios da educação do ser humano. Ela é atravessada por um conjunto de fatores que contribuem para tal como ela é desenvolvida pelos seus agentes participantes e pela comunidade à qual faz parte. A realidade e o contexto em que está inserida demarca sua estrutura e seu funcionamento. Ao mesmo tempo, se compõe por uma relação de convívio de classes sociais, culturas e valores diversos 
(OLIVEIRA; MARINHO-ARAÚJO, 2009). Em meio a isso, o psicólogo que atua na escola, deve se munir de diversos conhecimentos, para além da área da psicologia, visando que o trabalho ultrapasse os alunos-problema, mas possa atingir a todos, permitindo que os professores percebam seus alunos com um jeito individual, facilitando o desempenho de cada um (VALLE, 2003). Um dos fatores que dificultam a atuação do psicólogo na escola é a própria capacitação dos psicólogos, visto que nas universidades, os acadêmicos de psicologia nem sempre são preparados para as demandas em educação (EVANGELISTA; AMARAL, 2017). Dessa forma é crucial a formação contínua do profissional, bem como cursos que viabilizem o conhecimento aliado a prática profissional no espaço escolar.

Considerando que o espaço escolar é constituído por alunos, professores, famílias, funcionários, equipe diretiva e comunidade na qual está inserida, algumas dificuldades para a inserção do psicólogo no ambiente escolar se dão pelo desconhecimento do trabalho efetivo desse profissional. Ainda existe a marca clínica do psicólogo, não se reconhecendo o profissional como facilitador das relações interpessoais entre esses agentes escolares, assim como na relação ensino-aprendizagem. O psicólogo que irá atuar neste ambiente então, deverá criar espaço para escutar as demandas que emergem na escola e na educação como um todo e pensar em formas para lidar com essas demandas. Isso favorece a reflexão dentro da escola, com todos os agentes para que se possa trabalhar com essas relações e paradigmas (VALLE, 2003).

Em uma revisão bibliográfica sobre a saúde escolar da América Latina, Casemiro, Fonseca e Secco (2014) discutiram acerca do foco em torno a doença, triagem e prevenção, elucidando também que o direcionamento à promoção da saúde tem impulsionado mudanças. Ainda, achados importantes em referência a quantidade de publicações, mostrando o Brasil como o país que mais produziu artigos sobre o tema, comparativamente aos países da América Latina. Para solucionar os déficits em relação à saúde, os autores articulam sobre a importância de trabalhos interdisciplinares, desenvolvimento de políticas públicas e foco na promoção da saúde nos ambientes escolares, envolvendo comunidade escolar e diversos profissionais que atuam na área da saúde. Da mesma forma, Valle (2003) concorda que a saúde na escola deve ser aplicada envolvendo distintos conhecimentos, articulando com outras competências em detrimento da ética de transformação social e desenvolvimento da saúde mental.

Dentre exemplos das possibilidades de trabalho de um psicólogo atuando na escola de ensino fundamental, estudos brasileiros referem: o combate ao bullying e manejo de conflitos interpessoais (SANTOS, 2015), a promoção de saúde e desenvolvimento de habilidades sociais (GUIMARÃES; AERTS; CÂMARA, 2013), o desempenho acadêmico e relação com 
desenvolvimento social (CIA; COSTA, 2012), a psicologia institucional na escola e as relações de poder (QUEVEDO; MAGGI, 2018), a inclusão escolar de crianças com autismo e deficiência intelectual (BENITEZ; DOMENICONI, 2018), a educação sexual em contexto escolar (MOURA; PACHECO; DIETRICH; ZANELLA, 2011), as práticas de psicólogos escolares junto aos docentes das escolas públicas (AQUINO; LINS; CAVALCANTE; GOMES, 2015), entre tantos outros temas que podem ser abordados por um psicólogo que atue na escola.

Dentro desse contexto de possibilidade de atuação do psicólogo na escola, se faz necessário refletir sobre as práticas profissionais e as modalidades de trabalho exequíveis em ambiente escolar. A escolha pelo Ensino Fundamental se deu por este compreender anos iniciais escolares, ou seja, crianças em desenvolvimento cognitivo, social e biológico, sendo que a escola favorece esse desenvolvimento, especialmente na forma cognitiva (aprendizagem) e social (relacionamento entre pares e com professores, direção e outros agentes escolares). Dessa forma, o objetivo desse estudo foi de investigar a atuação do psicólogo na escola de ensino fundamental, pontuando as modalidades de trabalho que estão sendo executadas atualmente.

\section{Método}

Trata-se de uma revisão sistemática da literatura. Realizou-se uma busca nas bases de dados Biblioteca Virtual em Saúde (BVS), PubMed Central (PMC), Scielo, Directory of Open Access Journals (DOAJ) e Directory of Open Access Repositories (OpenDOAR), sendo considerados os seguintes descritores: psicologia, escola, relato de experiência, utilizando recursos avançados de pesquisa booleana com o recurso AND no cruzamento entre descritores. Os critérios de inclusão foram trabalhos publicados em língua portuguesa e inglesa, compreendendo o período entre 2014 e 2019, que abarcassem relatos de experiência de atuação de psicólogos que atuam em escolas de ensino fundamental. Após a busca, foi realizada a leitura dos resumos dos artigos, e para o relato e análise dos itens utilizou-se as recomendações do PRISMA (Principais Itens para Relatar Revisões sistemáticas e Meta-análises), de Moher et al. (2009).

\section{Resultados}

A busca inicial dos artigos evocou 93 resultados nas cinco bases de dados pesquisadas, sendo: 47 arquivos na BVS, dois na PMC, 14 na Scielo, 30 na DOAJ e zero na OpenDOAR. 
Diante esses resultados, foram analisados 57 resumos e cinco foram excluídos por duplicidade. Dessa forma, dos 52 resumos, foram excluídos 23 artigos por não se enquadrarem ao período de tempo utilizado como critério de inclusão para esse estudo (2014-2019), assim como outros 21 foram excluídos por não se enquadrarem na temática proposta para essa revisão. Restaram, dessa forma, oito artigos, os quais compõem a amostra dessa revisão sistemática. O processo de busca, seleção e distribuição dos artigos pode ser visualizado na Figura 1.

Figura 1 - Fluxograma de busca, seleção e distribuição dos artigos

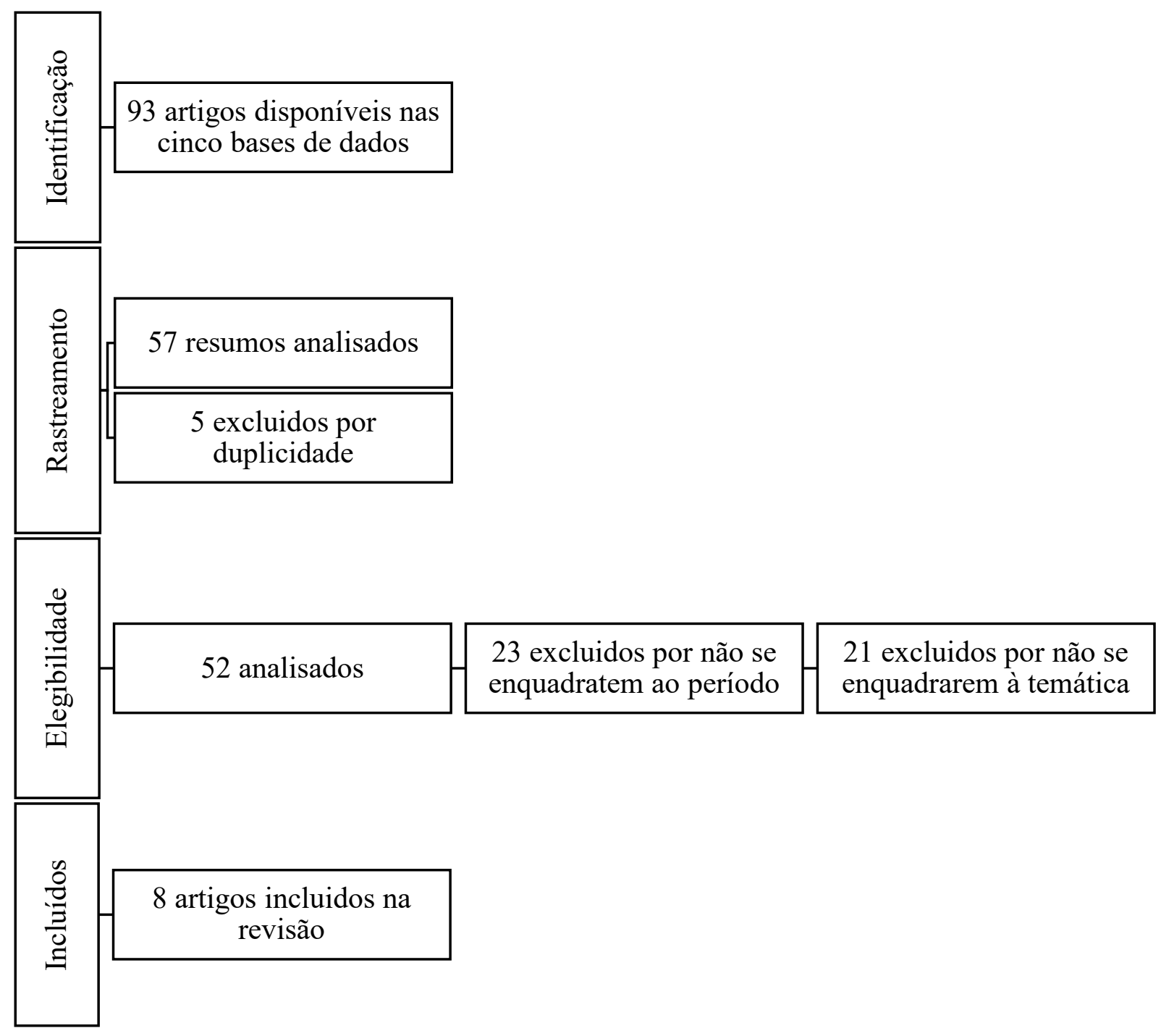

Fonte: Elaborado pela autora

Os artigos incluídos para essa revisão foram organizados na Tabela 1, com nomes dos autores e o título de cada trabalho. 
Tabela 1 - Artigos incluídos na revisão sistemática

\begin{tabular}{|c|c|}
\hline Autores & Título \\
\hline BULHÕES (2018) & $\begin{array}{l}\text { A construção de práticas críticas em espaços de formação do/a } \\
\text { psicólogo/a escolar }\end{array}$ \\
\hline MIURA et al. (2018) & $\begin{array}{l}\text { O ambiente escolar como espaço potencial para adolescente: } \\
\text { relato de experiência }\end{array}$ \\
\hline $\begin{array}{l}\text { OLIVEIRA; TAVEIRA; NEVES } \\
\text { (2014) }\end{array}$ & $\begin{array}{l}\text { Sensibilizar professores para o desenvolvimento de carreira } \\
\text { dos alunos: Relato de uma experiência }\end{array}$ \\
\hline PERINI et al. (2016) & $\begin{array}{l}\text { Relato de experiência sobre a reflexão da conduta passiva em } \\
\text { ambiente escolar }\end{array}$ \\
\hline QUEVEDO; CONTE (2017) & $\begin{array}{l}\text { Projeto defesa à vida: a psicologia na escola de ensino } \\
\text { fundamental }\end{array}$ \\
\hline $\begin{array}{l}\text { ROSE; BONDIOLI; AFONSO; } \\
\text { GONÇALVES; } \quad \text { PREZENSZKY } \\
(2016)\end{array}$ & $\begin{array}{l}\text { Práticas educativas inovadoras na formação do psicólogo } \\
\text { escolar: uma experiência com aprendizagem cooperativa }\end{array}$ \\
\hline $\begin{array}{l}\text { SAVEGNAGO; FARAJ; ARPINI; } \\
\text { SIQUEIRA (2015) }\end{array}$ & $\begin{array}{l}\text { Oficinas com meninas em uma escola aberta: espaço de } \\
\text { diálogo, reflexão e reconhecimento da singularidade }\end{array}$ \\
\hline SILVA; GANDA (2019) & $\begin{array}{l}\text { Intervenção em psicologia escolar: relato de experiência com } \\
\text { crianças de um projeto de educação integral }\end{array}$ \\
\hline
\end{tabular}

Fonte: Elaborado pela autora

Todos os artigos dessa amostra foram escritos em Língua Portuguesa, sendo que somente o estudo de Oliveira et al. (2014) foi realizado em Portugal, o restante foi composto por estudos com população brasileira (BULHÕES, 2018; MIURA et al., 2018; PERINI et al., 2016; QUEVEDO; CONTE, 2017; ROSE, et al., 2016; SAVEGNAGO, et al., 2015; SILVA; GANDA, 2019). Todos os estudos foram descritos a partir da metodologia de relato de experiência, sendo que em sua maioria foram realizados por estagiários do curso de Psicologia (BULHÕES, 2018; MIURA et al., 2018; QUEVEDO; CONTE, 2017; PERINI et al., 2016; ROSE, et al., 2016; SILVA; GANDA, 2019). Somente um trabalho foi realizado inteiramente por Psicólogos (OLIVEIRA, et al., 2014) e outro foi realizado por acadêmicos e profissionais formados em Psicologia, atuando em conjunto (SAVEGNAGO, et al., 2015).

Referente aos participantes dos estudos selecionados e intervenções realizadas, Bulhões (2018) realizou sua intervenção no período de 10 meses com três crianças do $1^{\circ}$ ano do Ensino Fundamental, encaminhadas pela escola com queixa escolar (dificuldades de aprendizagem), sendo que também foram acessadas as famílias, a partir de visitas domiciliares quinzenais, encontros com a professora das crianças e observação em sala de aula. Quevedo e Conte (2017) realizam a intervenção ao longo do período de um ano com alunos do Ensino Fundamental, na modalidade grupal, sendo um grupo reflexivo de 14 meninas com idade entre 13 e 16 anos, um 
grupo reflexivo de 10 meninos com idade entre 13 e 15 anos e outro grupo com seis adolescentes de ambos os sexos com idade entre 13 e 15 anos. As temáticas abordadas nos grupos foram sexualidade, bullying, agressividade, habilidades sociais, amizades, relacionamentos interpessoais e familiares, escola, entre outros. Além desses grupos, realizou-se um encontro de pais, no qual foi abordada uma postura psicoeducativa sobre os comportamentos dos filhos e sobre como os próprios pais vivenciaram sua fase da adolescência. Sobre os aconselhamentos individuais realizados com os alunos, destacou-se um espaço de escuta para atender a demandas pontuais, sendo que a principal demanda emergente se relacionava ao uso de drogas.

A intervenção de Rose et al. (2016) foi realizada com alunos do $2^{\circ}$ Ciclo do Ensino Fundamental ( $6^{\circ}$ a $9^{\circ}$ anos), com a participação total de 140 alunos. As intervenções foram em forma de grupos para turmas que apresentassem baixo aproveitamento acadêmico, dificuldade de adaptação ao contexto escolar e baixa motivação para atividades acadêmicas. Nesse estudo, os temas de interesse foram sexualidade na adolescência, preservação do meio ambiente, e fatores de risco ao uso de drogas. Silva e Ganda (2019) interviram com 14 crianças entre 8 e 10 anos de idade, que cursam entre o $3^{\circ}$ e $5^{\circ}$ ano do Ensino Fundamental. No período de nove meses, foram realizadas observações, seguidas pela prática de atividades e dinâmicas com os grupos de crianças, abordando temáticas como cooperação e comportamentos inadequados e vulnerabilidade social na escola. Miura et al. (2018) interviram com 26 alunos matriculados no $5^{\circ}$ ano do ensino fundamental com média de 11 anos de idade, usando o formato de sete oficinas de 60 minutos cada. Foram utilizados desenhos, contação de histórias, vídeos, colagens e dinâmicas, priorizando instrumentos lúdicos para trabalhar os seguintes temas: adolescência, gravidez na adolescência, abuso sexual e bullying.

Ainda, o trabalho de Savegnago et al. (2015), abordou uma intervenção realizada com meninas de 12 a 17 anos numa instituição que funciona como escola aberta. No período de um ano realizou-se oficinas na modalidade quinzenal com duração de $1 \mathrm{~h}$ e 30 minutos cada encontro. As oficinas decorreram da utilização de materiais lúdicos e audiovisuais, bem como dinâmicas de grupo, abordando temas como relações familiares, violência, sexualidade, em especial a gravidez na adolescência, já que existiam meninas com esse perfil, e focando também em projetos de vida para essas adolescentes.

Por outro lado, autores como Oliveira et al. (2014), realizaram uma intervenção de 90 minutos com 18 professores do ensino básico português $\left(2^{\circ}\right.$ e $3^{\circ}$ Ciclos $)$. Essa intervenção visou promover, junto dos professores, atitudes positivas quanto ao futuro, estimular o trabalho cooperativo na comunidade escolar e aproximar a escola de contextos laborais. A sessão teve como objetivos: esclarecer o conceito de desenvolvimento de carreira aos professores; 
conscientizar esses profissionais para o desenvolvimento de carreira dos alunos; e favorecer a condução de atividades com os professores para promover o desenvolvimento de carreira dos alunos. Perini et al. (2016), realizaram sua intervenção com 15 professores e 20 alunos do $9^{\circ}$ ano de uma escola pública de ensino fundamental. Com os alunos foram realizados encontros semanais de 45 minutos de duração. Com os professores foram realizados encontros grupais, nos quais foram discutidos diversos assuntos solicitados pelos participantes, promovendo um espaço de problematização de suas questões.

Sobre os resultados dessas intervenções, Bulhões (2018) ressaltou que as três crianças atendidas durante o estágio superaram as dificuldades de aprendizagem relacionadas à apropriação da escrita. Os familiares tornaram-se mais participativos na vida escolar das crianças e a instituição de ensino passou a oferecer espaços de participação da comunidade na escola. Miura et al. (2018) demonstraram que os participantes tiveram empenho nas atividades e expressaram suas opiniões com relação aos temas abordados. A realização da proposta possibilitou o acesso a uma forma potencializadora de vivenciar o ambiente escolar, bem como os temas que perpassam suas vivências cotidianas. Os autores descrevem resultados positivos em cada uma das oficinas realizadas com as diferentes temáticas, proporcionando formas diferentes de vivenciar sua participação na escola. Quevedo e Conte (2017) citam que seu trabalho reverberou em aspectos de atenuação de dificuldades, bem como a promoção da saúde psicológica dos alunos e pais que foram alvo das intervenções. Refere-se no artigo a resultados positivos frente às intervenções, especialmente no que remete à preservação da qualidade de vida na escola.

Sobre os resultados dos trabalhos que interviram com professores e direção, Perini et al. (2016) apontaram mudanças na perspectiva passiva da gestão para a contemplação de atitudes ativas diante das problemáticas do cotidiano escolar, ressignificando modelos instituídos da instituição. Os autores ainda referem que houve uma boa mudança em relação a direção e a coordenação, apresentando um posicionamento positivo para a solução de problemas emergentes. Oliveira et al. (2014) apontaram como resultados a valorização das experiências profissionais dos professores, assinalando a necessidade de aprofundar práticas promotoras do desenvolvimento de carreira para os alunos da escola, propiciando reflexões acerca das carreiras dos próprios professores.

Os autores Savegnago et al. (2015) apontaram resultados positivos, em que as oficinas se constituíram como momentos reflexivos sobre as condições de vida presente das adolescentes, bem como pensando em planos para o futuro, com a implementação de pensar sobre projetos de vida. As autoras afirmam que ações como esta possibilitam esclarecimento às 
adolescentes para pensar na construção de planos saudáveis e que envolvam crescimento emocional. As intervenções realizadas possibilitam ainda uma boa vinculação com as coordenadoras dos grupos e as adolescentes, o que favoreceu a troca de experiências e o espaço necessário para trabalhar temas importantes marcados por um período de vida instável da adolescência e de conflitivas familiares e sociais.

O estudo de Rose et al. (2016) apontou resultados positivos significativos, como uma boa interação entre os vínculos afetivos com os alunos e os coordenadores dos grupos. Trabalhou-se com habilidades sociais, sendo estas necessárias para o desenvolvimento dos grupos, bem como na cooperação de aprendizagem para o sucesso do grupo. Destaca-se que houve o encorajamento para que todos participassem do grupo, ouvir os colegas, resolver conflitos, esperar a vez de falar, por exemplo. O grupo se motivou com os feedbacks, o que contribuiu para o empenho e aprendizagem de todos. Os autores ainda discutem a importância da escola como espaço de desenvolvimento social e acadêmico, e que essas experiências favorecem vivências saudáveis e estratégicas para a vida como um todo. Mesmo assim, um ponto importante destacado nesse estudo, alguns adolescentes se favoreceram mais das intervenções enquanto outros precisarão de mais oportunidades de aprimoramento de habilidades para um melhor resultado.

Por outro lado, Silva e Ganda (2019) apenas mencionaram resultados em relação ao desenvolvimento da estagiária, não desenvolvendo no que as intervenções realizadas contribuíram para a escola e para com os diferentes atores escolares. Se faz importante destacar as contribuições dessa intervenção, visando contribuir com outros estagiários e profissionais que atuam na escola. Porém, compreende-se que o objetivo do artigo foi destacar o desenvolvimento da estagiária nesse momento.

\section{Discussão}

O objetivo dessa revisão sistemática foi investigar a atuação do psicólogo na escola de ensino fundamental, pontuando as modalidades de trabalho que estão sendo executadas atualmente. De acordo com os resultados, Quevedo e Conte (2017) e Miura et al. (2018), apresentaram um trabalho com intervenções na modalidade grupal voltadas ao desenvolvimento emocional e psicoeducativo com alunos, relacionando, em ambos estudos, temas pertinentes da adolescência, como por exemplo, sexualidade, bullying, relações interpessoais e habilidades sociais. Ainda, ambos estudos afirmaram resultados positivos para os alunos e para o funcionamento da escola. 
Diante essas possibilidades de trabalho, percebe-se a necessidade desse profissional atuando na escola, pontuando um espaço de desenvolvimento emocional saudável. Ainda, destaca-se a importância de que as intervenções sejam descritas com aprofundamento, apontando materiais que foram utilizados, dinâmicas, modelos pré-existentes de conteúdos e base teórica que dá suporte ao entendimento dinâmico da Instituição e dos atores da instituição que foram alvo das intervenções (alunos, professores, funcionários, equipe diretiva, família).

Os artigos utilizados nessa revisão sistemática refletem uma descrição detalhada das intervenções, bem como dos que foram alvo da intervenção e de quem coordenou as atividades nas escolas. Torna-se valioso destacar a formação dos profissionais envolvidos na criação das atividades e dinâmicas, nas escolhas de materiais e como o público-alvo foi inserido nas atividades, sua participação ou dificuldades decorrentes do processo.

Poder compartilhar os pontos positivos e também negativos do processo de aceitação dentro da instituição, assim como da intervenção em si valoriza o trabalho do profissional da psicologia, visto que nem sempre há uma boa aceitação por parte da Instituição ou dos que irão receber as intervenções. Podem haver conflitos e resistências, pontos estes que devem ser argumentados conforme a literatura e as possiblidades dentro da instituição. Cada item e cada tema descrito nos artigos que compuseram essa revisão servem de base para que outros profissionais, sejam eles estagiários ou graduados, possam se identificar para que os próximos artigos que sejam escritos venham a fazer uso do conhecimento que essas fontes podem proporcionar aos psicólogos que vão atuar na Escola de Ensino Fundamental.

\section{Considerações finais}

As possibilidades de atuação do psicólogo na escola de ensino fundamental são diversificadas. Porém, considerando a pouca produção de artigos com a temática analisada, destaca-se um nicho de pesquisa ainda a ser melhor desenvolvido. Somente um dos artigos foi realizado com profissionais formados em Psicologia, sendo que os demais são relatos de experiência de estágio de estudantes de Psicologia. Uma possível hipótese sobre esse resultado seria de que profissionais perdem interesse na publicação de relatos de experiência, uma vez que se voltam para a prática propriamente dita ou para pesquisas que envolvam uma metodologia mais rigorosa, como por exemplo, pesquisas empíricas. Uma possível limitação, a metodologia de relato de experiência é pouco valorizada em revistas de publicação nacional, sendo de difícil a aceitação para publicação deste tipo de estudo. Dessa maneira, os profissionais sentem-se desmotivados a realizar esse tipo de publicação. 
Dentre as limitações desse estudo, destaca-se a quantidade de artigos incluídos, bem como a única forma de acesso às bases de dados foi em sua forma livre, ou seja, não se utilizou bases de dados pagas e estas poderiam conter um número maior de resultados. Além disso, todos os artigos incluídos foram escritos em língua portuguesa, o que limita fazer uma discussão pautada em diferenças culturais em relação a atuação, intervenções, demandas e necessidades entre um país ou outro. Salienta-se ainda que limitar a pesquisa nos últimos cinco anos também gera uma restrição à quantidade de publicações, que poderiam ser em maior número caso a proposta fosse, por exemplo, incluir artigos dos últimos dez anos.

Sugere-se para futuras pesquisas vinculadas à temática, que seja posto em destaque as limitações advindas desse estudo, realizando revisões que contemplem aspectos que esta não se propôs a contemplar. Além disso, sugere-se aos psicólogos que atuam em escolas de ensino fundamental que possam redigir seus trabalhos, para que o desenvolvimento desse trabalho seja acessado por demais profissionais interessados na mesma área. A contribuição científica de relatos de experiência é de grande valia para profissionais, alunos e pessoas que tem interesse em conhecer a prática do trabalho do psicólogo em contexto educacional.

\section{REFERENCIAS}

AQUINO, F. S. B.; LINS, R. P. S.; CAVALCANTE, L. A.; GOMES, A. R. Concepções e práticas de psicólogos escolares junto a docentes de escolas públicas. Revista quadrimestral da Associação Brasileira de Psicologia Escolar e Educacional, v. 19, n. 1, p. 71-78, 2015.

BARBOSA, R. M.; MARINHO-ARAÚJO, C. M. Psicologia escolar no Brasil: reflexões e reflexões históricas. Estudos de Psicologia, v. 27, n. 3, p. 393-402, 2010.

BENITEZ, P.; DOMENICONI, C. A atuação do psicólogo na inclusão escolar de estudantes com autismo e deficiência intelectual. Psicologia Escolar e Educacional, v. 22, n. 1, p. 163$172,2018$.

BULHÕES, L. F. A construção de práticas críticas em espaços de formação do/a psicólogo/a escolar. Psicologia Escolar e Educacional, v. 22, n. 1, p. 211-213, 2018.

CASEMIRO, J. P.; FONSECA, A. B. C.; SECCO, F. V. M. Promover saúde na escola: reflexões a partir de uma revisão sobre saúde escolar na América Latina. Ciência e Saúde Coletiva, v. 19, n. 3, p. 829-840, 2014.

CIA, F.; COSTA, C. S. L. Desempenho acadêmico nas séries do ensino fundamental: Relação com o desenvolvimento social. Psicologia Argumento, v. 30, n. 68, p. 109-118, 2012.

EVANGELISTA, A. L. F.; AMARAL, A. F. Atuação do psicólogo escolar na rede pública de ensino, da teoria à prática: uma revisão bibliográfica conceitual. Psicologia e Saúde em Debate, v. 3, n. 2, p. 62-73, 2017. 
GUIMARÃES, G.; AERTS, D.; CÂMARA, S. G. A escola promotora da saúde e o desenvolvimento de habilidades sociais. Diaphora: Revista da Sociedade de Psicologia do Rio Grande do Sul, v. 12, n. 2, p. 88-95, 2013.

MIURA, P. O. et al. O ambiente escolar como espaço potencial para adolescente: relato de experiência. Revista Pesquisas e Práticas Psicossociais, v. 13, n. 2, p. 14-14, 2018.

MOHER, D.; LIBERATI, A.; TETZLAFF, J.; ALTMAN, D. G. The PRISMA Group. Preferred Reporting Items for Systematic Reviews and Meta-Analyses: The PRISMA Statement. PLoS Med, v. 6, n. 7, 2009.

OLIVEIRA, C. B. E.; MARINHO-ARAÚJO, C. M. Psicologia escolar: cenários atuais. Estudos e pesquisas em psicologia, v. 9, n. 3, p. 648-663, 2009.

OLIVEIRA, Í. M.; TAVEIRA, M.; NEVES, L. F. Sensibilizar professores para o desenvolvimento de carreira dos alunos: Relato de uma experiência. Psicologia Ciência e Profissão, v. 34, n. 2, p. 512-523, 2014.

PERINI, J. P. et al. Relato de experiência sobre a reflexão da conduta passiva em ambiente escolar. RELACult-Revista Latino-Americana de Estudos em Cultura e Sociedade, v. 2, n. 4, p. 134-147, 2016.

QUEVEDO, R. F.; MAGGI, A. Por uma psicologia institucional na escola: reflexões acerca das relações de poder. In: CLAUS, S. M.; STEDILE, N. L. (Org.). Rede de Cuidados em Saúde. 1. ed. Caxias do Sul: Traço diferencial, 2018. v. 1, p. 169-184.

QUEVEDO, R. F.; CONTE, R. F. Projeto defesa à vida: a psicologia na escola de ensino fundamental. Psicologia: Teoria e Pesquisa, v. 32, n. 2, 2017.

ROSE, T. M. S. et al. Práticas educativas inovadoras na formação do psicólogo escolar: uma experiência com aprendizagem cooperativa. Psicologia: Ciência e Profissão, v. 36, n. 2, p. 304-316, 2016.

SAVEGNAGO, S. D. O. et al. Oficinas com meninas em uma escola aberta: espaço de diálogo, reflexão e reconhecimento da singularidade. Temas em Psicologia, v. 23, n. 2, p. 467-480, 2015.

SILVA, F. C.; GANDA, D. R. Intervenção em psicologia escolar: relato de experiência com crianças de um projeto de educação integral. Psicologia e Saúde em debate, v. 5, n. 1, p. 37 49, 2019.

SANTOS, D. L. Contribuições da psicologia escolar para a prevenção e combate ao bullying. Diaphora: Revista da Sociedade de Psicologia do Rio Grande do Sul, v. 15, n. 2, 2015.

VALLE, L. E. L. R. Psicologia escolar: um duplo desafio. Psicologia Ciência e Profissão, v. 23, n. 1, p. 22-29, 2003. 


\section{Como referenciar este artigo}

QUEVEDO, R. F. A atuação do psicólogo na escola de ensino fundamental: modalidades de trabalho. Doxa: Rev. Bras. Psico. e Educ., Araraquara, v. 22, n. 2, p. 381-393, jul./dez. 2020. e-ISSN: 2594-8385. DOI: https://doi.org/10.30715/doxa.v22i2.13811

Submetido em: 27/03/2020

Revisões requeridas: $17 / 07 / 2020$

Aprovado em: 17/07/2020

Publicado em: 30/08/2020 